\title{
Sensitive defects
}

Single-walled carbon nanotubes (SWNTs)

can be used to detect a number of chemical vapours. The presence of vapour is determined from pronounced changes that occur in the electronic properties of the SWNTs - conductance, capacitance and so on — as the gas molecules interact with their surface. Previous studies have presumed that this interaction takes place between the vapour molecules and the 'pristine' walls of the nanotube. Joshua Robinson and colleagues now report (Nano Letters 6, 1747-1751; 2006) the use of a combination of experimental data and electronic calculations to show that molecules such as acetone and methanol binding to defects in the surface of SWNTs have a much greater effect on the electrical properties of the nanotube than those binding at other points on the length of the tube.

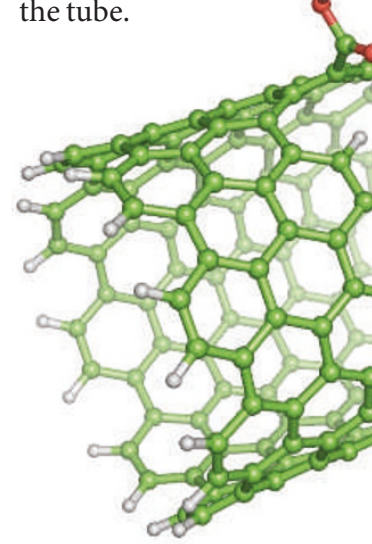

The authors demonstrate that an increase in the number of defects present in

the structure of SWNTs greatly enhances their electronic response to gas sorption. This large response is due to the increased binding energy available at the

\section{FUNCTIONAL FILMS}

Nanoporous thin films have potential uses in many applications, ranging from antireflective films, through sensors and membranes, to electronic devices. A fabrication technique that allows more flexibility and tunable optical properties than previously offered is now reported by Cho et al. (J. Am. Chem. Soc. 128, 9935-9942; 2006). By preparing nanoporous films by self-assembly of blockcopolymer micelles, they can control the level of porosity by simply varying the solution pH or polymer block sizes, and thereby produce antireflective films without any post-processing steps. Moreover, as hydrophobic molecules can be incorporated in the micelle cores, an encapsulated water-insoluble dye provides films that are not only antireflective, but can also be optically switched to transmit different colours. The technique is simpler than other methods to introduce porosity in polymer films via post-fabrication treatment such as etching, and provides additional flexibility as compared with the self-assembly of other building blocks, which only allows the introduction of hydrophilic guest molecules. The possibility to incorporate a wide range of other functional guest molecules within the micelle interiors makes these films ideally placed for use in an extended range of applications.

A touch-up tool for light traps

A semiconductor nanocavity is an artificially patterned structure that effectively traps light with a precise wavelength within a nanometre volume. The resonance wavelength is determined by both the semiconductor properties and the geometry of the cavity, and can be predicted very accurately. Once the cavity is fabricated though, it is hard to change its geometry to even slightly vary the resonance wavelength. Kevin Hennessy and colleagues have found the solution by working on the semiconductor instead (Appl. Phys. Lett. 89, 041118; 2006). With an atomic force microscrope tip they managed to oxidize very specific areas within a GaAs cavity. As the optical properties of the oxide are different form those of the pure GaAs, they were able to tune the resonance wavelength with very high precision, and over a range of several nanometres, by varying the oxidation area. These results could be very important for applications in quantum information processing that require tuning the cavity photons in perfect resonance with the light emitted by semiconductor nanostructures (typically quantum dots) embedded in the cavity.

\section{Smart liquid lenses}

A group of researchers in the US have developed a liquid lens that can focus automatically, controlled by a stimuli-responsive hydrogel (L. Dong et al. Nature 442, 551-553; 2006). A ring of hydrogel sits in a water-filled microfluidic channel, and is covered with a glass slide having a central aperture. Oil is poured on top of this, and is then capped off with a complete glass slide. The interface between the oil and the water forms the liquid lens, which is held in place due to the fact that the upper surface of the aperture slide is hydrophobic, and the lower surface is hydrophilic.
When a stimulus such as $\mathrm{pH}$ or temperature change is applied to the water channel, the hydrogel swells or shrinks, altering the volume of the water droplet held within it, which in turn alters the curvature, and thus the focal length, of the lens. The flexibility of this system the hydrogel can be shrunk to $1 \mathrm{~mm}$ across, can be of different shapes, and can be chemically tuned to respond to various stimuli - makes it attractive for applications such as light sensing, or biomedical imaging, or even, sometime in the future, as a replacement lens for the human eye.

\section{Periodicity in the woodpile}

A three-dimensional photonic crystal with two different periodicities has been fabricated by Yan and colleagues in Singapore (Langmuir 22, 7001-7006; 2006). In photonic crystals - artificially created periodic structures of dielectric material - light propagates in a fashion similar to that of electrons moving within a semiconductor. In particular, the presence of the so-called photonic bandgap forbids the propagation of light of wavelength within a precise range. The size of the bandgap varies with the structural periodicity and the dimension of the periodic arrangement. So far, only relatively simple structures with the same periodicity in every direction have been produced, using various techniques. Yan et al. have now succeeded in producing a structure with different periodicities by combining the two main methods of preparing photonic crystals: a lithographic technique, with which they produced colloidal crystal strips having a face-centredcubic structure giving one periodicity, and a self-assembly technique, in which the crystal strips are stacked layer by layer in a woodpile structure to give a second periodicity. It is thought that these new structures will have many potential applications - as three-dimensionally connected waveguards, sensors and superprisms, for example.

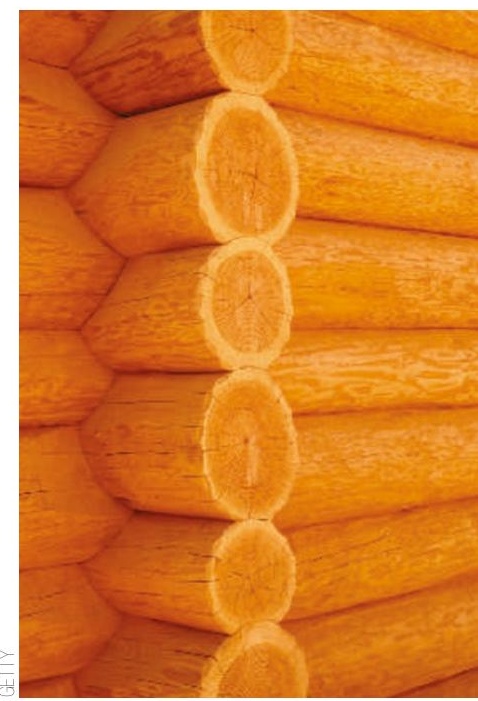

